\title{
Lattice Boltzmann simulation for flux change under oscillating boundary condition
}

\author{
Ryuta UEDA $^{1}$, Hitoshi MIKADA ${ }^{1}$, Tada-nori GOTO ${ }^{1}$ and Junichi TAKEKAWA ${ }^{1}$ \\ ${ }^{1}$ Dept. of Civil and Earth Res. Eng., Kyoto University
}

Seismic stimulation is known as one of the methods of Enhanced Oil Recovery (EOR). Numerous observations show that seismic stimulation of oil reservoir may alter water and oil production. Recent studies attempted to show detailed mechanisms of seismic stimulation. But understanding the detail mechanisms, it is necessary to delineate the relationship between macroscopic and microscopic phenomena So, to use seismic EOR efficiently, we attempt to understand amplitude characteristics and frequency characteristics of the flux change in viscous laminar flow under oscillating boundary condition to simulate seismic EOR. In this time, we start to analyze a single-phase flow in various pore shapes and scales with 2D LBM scheme. The results show seismic amplitude, frequency, pore scales, shapes and angle are largely related with the flux change, because of the reduction of pressure loss and the flow velocity difference between the wall and flow.

\section{Introduction}

The amount of oil production in the world is decreasing recently and it is of importance to seek the technological development for enhancing the recovery of oil in place in the subsurface: Enhanced oil recovery(EOR). EOR can be achieved by several methods: water injection, gas injection, chemical injection, and thermal injection. Seismic stimulation is also known as one of the methods of EOR. Many observations show that seismic stimulation of oil reservoir may alter water and oil production, because of altering the apparent viscosity coefficient, or contributing to the coalescence or dispersion of oil droplets. Clamen and Minton (1972) showed flow velocity under moving pipes harmonically was lower than one under non-moving case. Pride et al. (2008) attempted to simulate the mechanism of coalescence or dispersion of oil droplets with Lattice Boltzmann method (LBM). Beresnev (2010) attempted to analyze the phenomenon of oil droplet forced by seismic stimulation at the pore-throat. Zaaem et al. (2009) attempted to grasp the flux change of non-Newtonian fluid under vibration. These studies are aimed to understand the mechanisms of oil and water production increment caused by seismic stimulation. But, for utilizing seismic EOR efficiently, we need to understand the relationship between the physical properties of rock and amplitude and frequency of the incident seismic waves.

In this study, we attempt to demonstrate the flux change in viscous laminar flow under oscillating boundary condition for the simulation of interstitial flow. Here, we analyze a single-phase flow in various pore scales and shapes with a $2 \mathrm{D}$ LBM scheme to discuss the possibility of flux change by pore shapes and scales.

\section{Method}

In this study, we analyze 2D incompressible flow. In incompressible viscous flow, the governing equation is Navier-Stokes equation (2a) and mass conservation equation $(2 b)$,

$$
\begin{gathered}
\rho \frac{\partial u_{i}}{\partial t}+\rho u_{j} \frac{\partial u_{i}}{\partial x_{j}}=-\frac{\partial p}{\partial x_{i}}+\mu \frac{\partial^{2} u_{i}}{\partial x_{j}^{2}} \\
\frac{\partial u_{i}}{\partial x_{i}}=0
\end{gathered}
$$

where $\rho$ is the fluid density, $u$ is the fluid velocity, and $\mathrm{p}$ is the fluid pressure.

CFD has some numerical methods like FDM, FEM and particle methods. In this study, we use Lattice Boltzmann method (LBM) described by Boltzmann equation. We use a 2-dimensional 9-velocity $(2 \mathrm{~d} 9 \mathrm{v})$ model to simulate 2-dimensional incompressible viscous flow (figure.1) The square lattice LBGK model is expressed as:

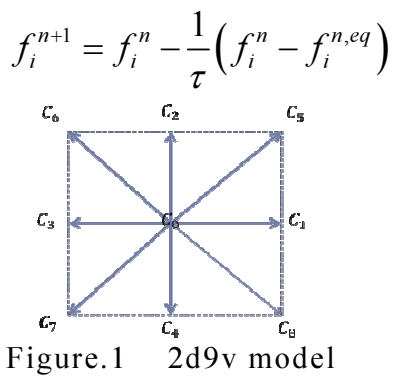


where second term of the right side is collision term. $f_{i}^{n, e q}$ is the equilibrium distribution function, $\tau$ is the relaxation coefficient which controls the rate of approach to equilibrium. The density per node, $\rho$ and the macroscopic flow velocity, u $=(\mathrm{Ux}, \mathrm{Uy})$, are defined in the terms of the particle distribution function by

$$
\sum_{i=0}^{8} f_{i}=\rho, \quad \sum_{i=0}^{8} f_{i} \cdot c_{i}=\rho \mathbf{v}
$$

The equilibrium distribution functions $f_{i}^{n, e q}$ depend only on local density and velocity and they can be chosen in the following form (2e).

$$
f_{i}^{n, e q}=w_{i} \rho\left(1+3 \mathbf{c}_{\mathrm{i}} \cdot \mathbf{u}+\frac{9}{2}\left(\mathbf{c}_{\mathrm{i}} \cdot \mathbf{u}\right)^{2}+\frac{3}{2} u^{2}\right)
$$

$\tau_{k}(2 \mathrm{f})$ is relaxation coefficient, and this is defined in the terms of kinematic viscosity coefficient $v$, time step $\mathrm{dt}$, and divider width $\mathrm{dx}$.

$$
\tau=\frac{3 d t}{d x^{2}}+\frac{1}{2}
$$

$w_{i}$ is a weighting function. Values of the weighting function in each directions are defined by

$$
w_{i}=\frac{4}{9}(i=0), \frac{1}{9}(i=1,2,3,4), \frac{1}{36}(i=5,6,7,8)
$$

We assume the solid is rigid. So, we add the internal force instead of oscillating boundary wall. In our simulation, we adopt the boundary conditions of Inamuro(1996).

Pore throats in rock have complex shape. Choi and Clayton(2000), Quadrio and Sibilla(2000), and Bewley(2001) showed span wise or stream wise oscillation may reduce the drag reduction and
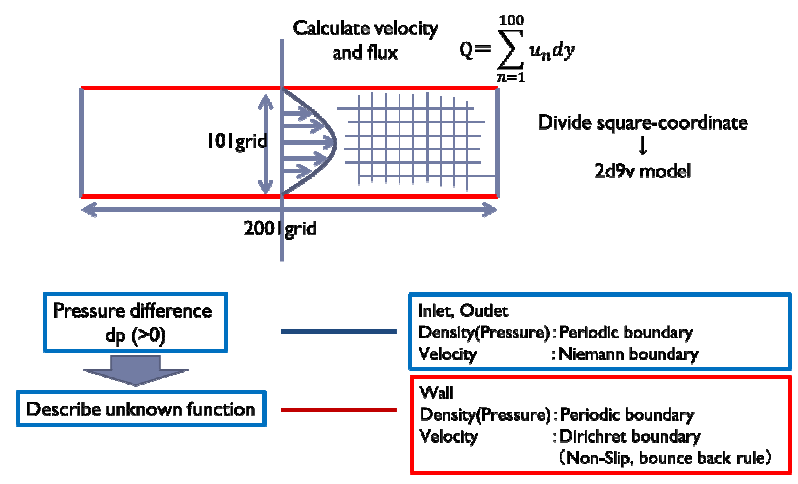

Figure. 2 boundary condition

(1) Sudden expansion, rapid shrinkegge model (3)/erallel panel model (scale large/sma I)

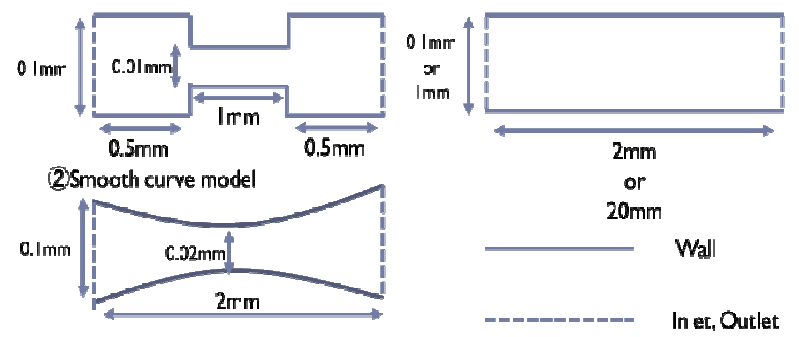

Figure. 3 Simulation shape improve flow. So, we attempt to simulate the flow under various pore shapes to grasp the influence of the pore shapes (Figure.3).

\section{Result}

In this study, we discuss five characteristics: amplitude, frequency, pore size, pore aspect ratio and incident angle. In these models, the pressure difference between inlet and outlet is $0.0098(\mathrm{~N})$.

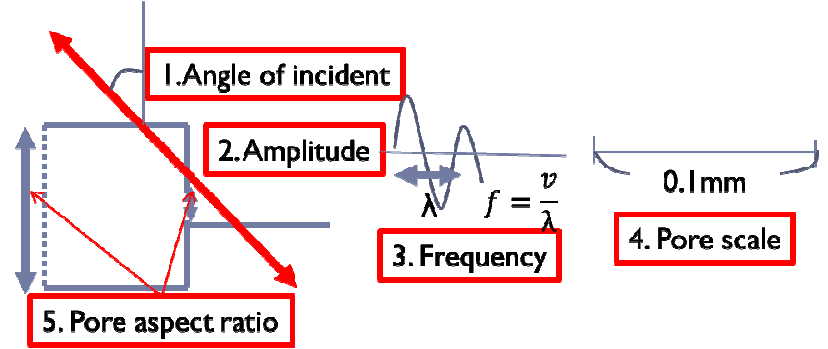

Figure. 4 Five different characteristics

\section{(1) Angle of Incident}

First, we discuss whether angles of incident of seismic wave cause the amount of flux change. Figure. 5 shows that the flux change of oscillating wall in horizontal direction is larger than that in vertical one. Vertical axis is the flux ratio between with and without the internal force.

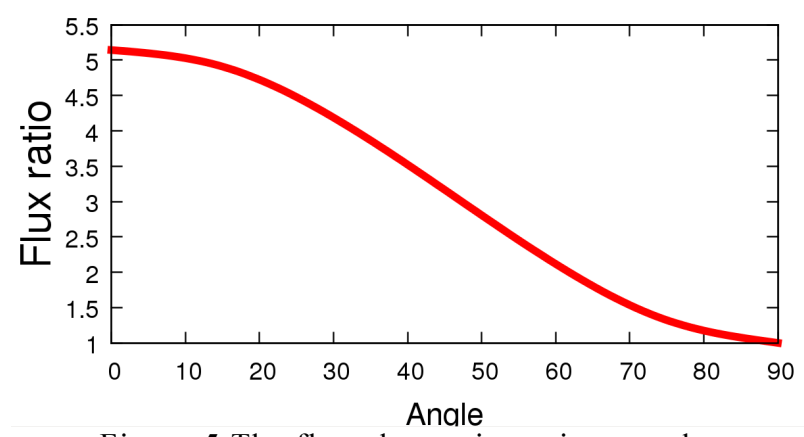

Figure. 5 The flux change in various angles

\section{(2) Amplitude characteristics}

Second, we discuss amplitude characteristics of flux increment. Figure. 6 shows that oscillating wall in horizontal direction may cause the flux reduction in large parallel panel model. In sudden expansion and rapid shrinkage model and smooth curve model, figure.6 also shows oscillating wall in horizontal direction may cause the flux increment. These figures also show that flux change in high amplitude oscillation is larger than that in low one. This indicates that the horizontal oscillation has the effect on the flux rate. On the contrary, figure.7 shows that oscillating wall in vertical direction may not cause the flux reduction and increment much. Also, in small parallel panel model, figure.6 shows that oscillating wall in horizontal direction may not cause flux increment and reduction. So, the angles of oscillation and pore size are related with the 
effect of flux increment and reduction. So, seismic stimulation under high seismic amplitude may be effective to flow in each pore throat.

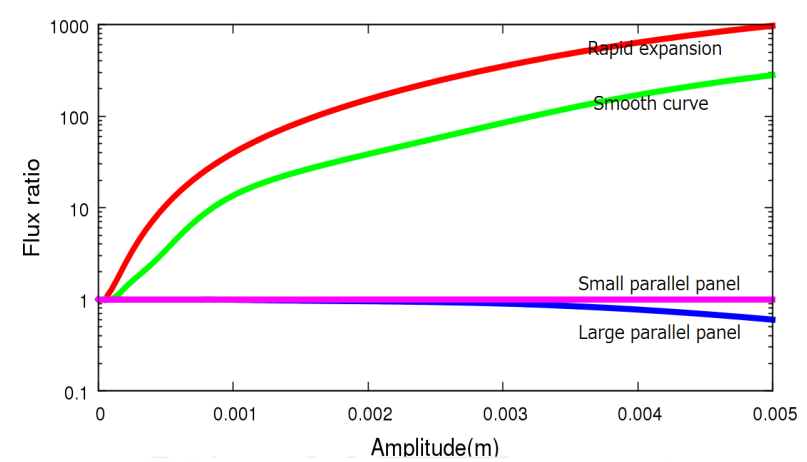

Figure.6 The flux change in horizontal oscillation

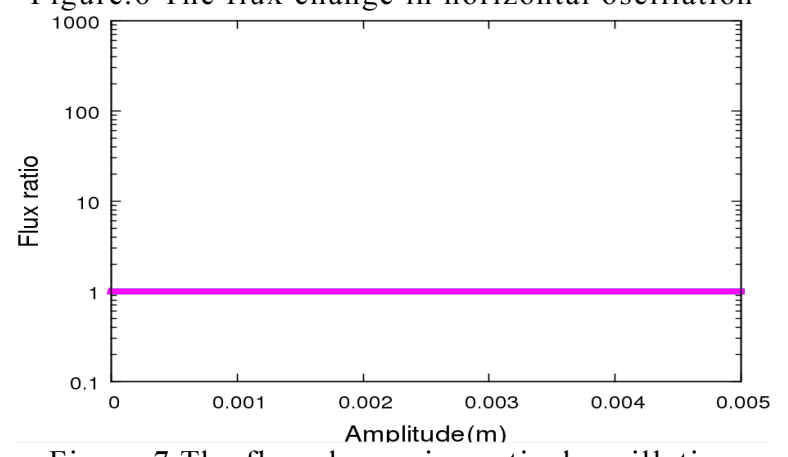

Figure. 7 The flux change in vertical oscillation

\section{(3) Frequency characteristics}

Third, we discuss frequency characteristics of flux increment. Figure. 8 shows that oscillating wall in horizontal direction has little effect to change flux in large parallel panel model. On the other hand, in sudden expansion and rapid shrinkage model and smooth curve model, figure. 8 also shows oscillating wall in horizontal direction may cause the flux increment. These figures also show that flux change in high frequency oscillation is larger than that in low one. So, horizontal oscillation also has the larger effect on the flux rate with different frequencies. On the contrary, figure. 9 shows that oscillating wall in vertical direction may not cause the flux reduction and increment much. Also, in small parallel panel model, figure.6 show that oscillating wall in horizontal direction may not cause flux change. So, the angles of oscillation and

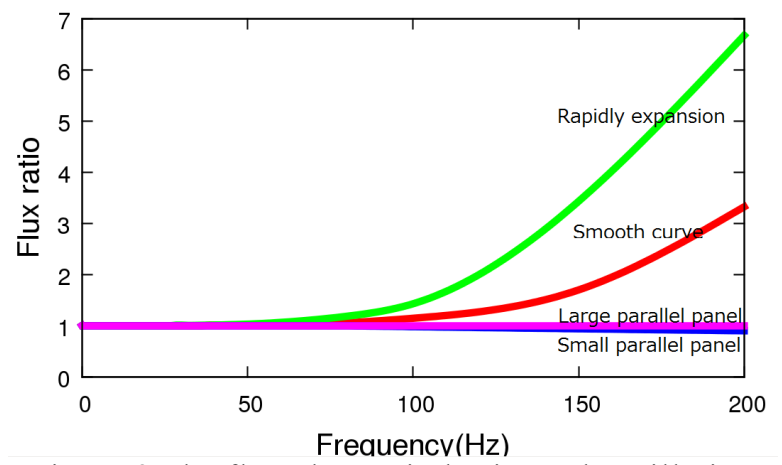

Figure. 8 The flux change in horizontal oscillation

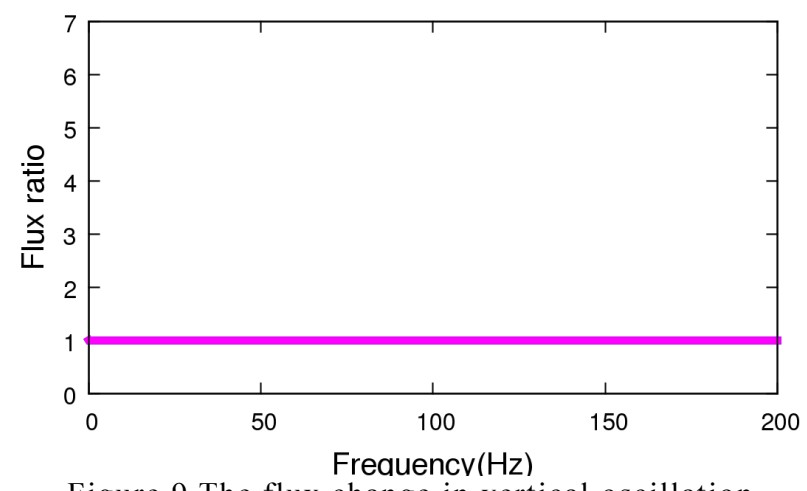

Figure.9 The flux change in vertical oscillation pore size are related with the effect of flux change.

\section{(4) Pore throat aspect ratio}

Last, we discuss the difference between large and small pore throat aspect ratio: large pore throat width to small pore throat width. Red line is the ratio 5 to 2 . Green line is the ratio, 10 to 1 . Comparing these two different pore throats, flux change in large pore aspect ratio is smaller than that in small one under low frequency zone. On the other hand, flux change in large pore aspect ratio is larger than that in small one under high frequency zone. So, in high permeability zone, low frequency seismic stimulation may be effective. But in low permeability zone, only high frequency seismic

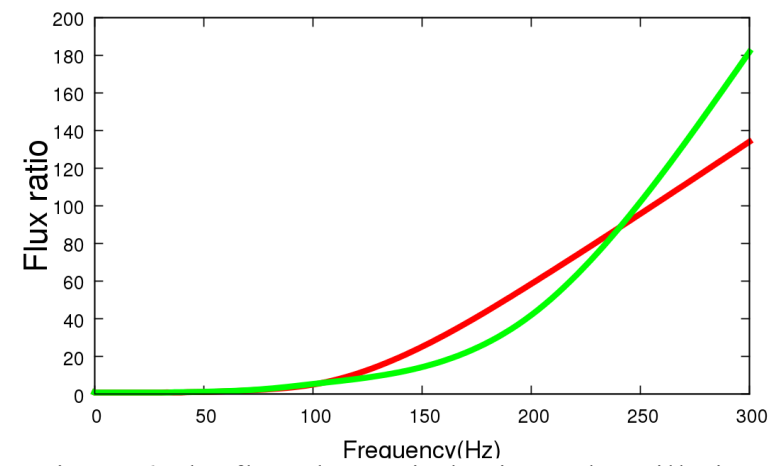

Figure. 6 The flux change in horizontal oscillation stimulation may be effective.

\section{Discussion}

We consider three reasons to change the flux: increment or reduction.

First, by large streamwise oscillation, large velocity difference between the wall and the flow central is generated. In low Reynolds flow, the horizontal wall oscillations propagate in fluid instantly. But if the time delay of propagation is generated, the flow resistance becomes higher than that without oscillating. So, the central velocity of flow under oscillating boundary is lower than under without oscillating.

Second, partial pressure gradient is generated by large spanwise oscillation. The vertical velocity difference between the flow and the wall cause the 
concentration / dispersion of fluid. If the oscillation generates the concentration of fluid, the fluid flow out in stream direction.

Finally, oscillating boundary causes improving pressure loss. Choi and Clayton (2000), Quadrio and Sibilla (2000), and Bewley (2001) suggest that wall oscillation cause the possibility of flux increment, because of drag reduction and pressure loss. In this simulation, sudden expansion, rapid shrinkage zone and small pore throat generate pressure loss. So, in this model, the oscillation

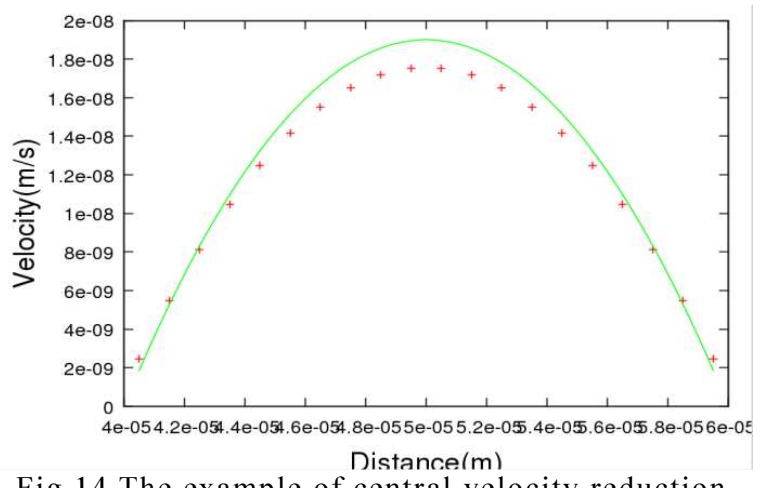

Fig. 14 The example of central velocity reduction (Sudden expansion and rapid shrinkage model)

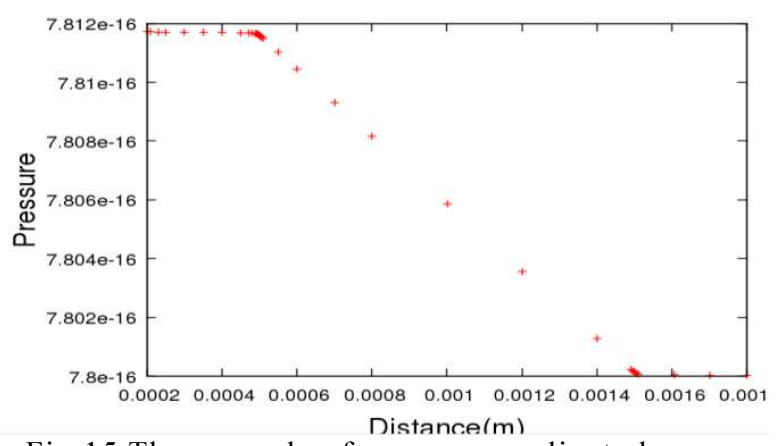

Fig.15 The example of pressure gradient change (Sudden expansion and rapid shrinkage model)

causes the possibility of large flux increment.

\section{Conclusion}

In this study, we discuss the possibility of flux change due to pore scales or shapes under oscillating boundary condition with LBM.

In consequence, sudden expansion and rapid shrinkage model generates larger flux change than that from the parallel panel model.

For next stage, we try to evaluate following three points. First, we attempt to consider two-phase flow. In two-phase flow, the oscillating boundary conditions generate the reduction of interfacial tension, improvement of relative permeability and the coalescence or dispersion of oil droplets. The consideration of effect of the volumetric change in the pore throat under $\mathrm{P}$ wave is also important. Finally, we attempt to simulate realistic pore throat model created by a Gaussian Random Field model.

\section{REFERENCES}

1) Beresnev, I.A. and Paul A.J. (1994) Elastic-wave stimulation of oil production: A review and results, Los Alamos National Laboratory, Geophysics, 59, 6, 1000-1017.

2) Beresnev, I.A., (2006) Theory of vibratory mobilization on nonwetting fluids entrapped in pore constrictions, Geophysics, 71, 6, N47-N56.

3) Beresnev, I.A, (2009) Viscosity effect in vibratory mobilization of residual oil, Geophysics, 75, 4, N79-N85

4) Clamen, M. and Minton, P., (1976) An experimental investigation of flow in an oscillating, J. Fluid Mech., 81, 421-431.

5) Kwing-So, C. and Brian, R. C., (2000) The mechanism of turbulent drag reduction with wall oscillation, International Journal of Heat and Fluid Flow, 22, 1-9

6) Maurizio, Q. and Stefano, S.(2000) Numerical simulation of turbulent flow in a pipe oscillating around its axis, J.Fluid Mech.,424, 217-241

7) Thomas, R. B., (2001) Flow control: new challenges for a new Renaissance, Progress in Aerospace Science, 37, 21-58

8) Pride, S.R., Flekkoy, E. and Aursjo, O., (2008) Seismic stimulation for enhanced oil recovery, Geophysics, 73, O23-P35.

9) Zaaem, M.A., Lapin, S., Matveev, K., (2009) The Effect of Vibration on Flow Rate of Non-Newtonian Fluid, Proc. Fourth SIAM Conference on Mathematics for Industry, 137-141. 